Investigación Descriptiva, Correlacional o Cualitativa

Pensar en Movimiento:

Revista de Ciencias del Ejercicio y la Salud ISSN 1659-4436

Vol. 14, No.2, pp. 1- 16

Cierre al 31 de diciembre, 2016
PENSAR EN

REVISTA DE CienCIAS DEL EJERCICIO Y LA SALUD

\title{
ANÁLISIS EXPLORATORIO Y COMPARATIVO DE LOS NIVELES DE ACTIVIDAD FÍSICA EN SESIONES DE MOTRICIDAD EN EDUCACIÓN INFANTIL
}

\author{
EXPLORATORY AND COMPARATIVE ANALYSIS OF THE PHYSICAL ACTIVITY \\ LEVELS ON MOTOR PERFORMANCE IN CHILDREN
}

\section{ANÁLISE EXPLORATÓRIA E COMPARATIVA DOS NÍVEIS DE ATIVIDADE FÍSICA EM SESSÕES DE MOTRICIDADE EM EDUCAÇÃO INFANTIL}

\author{
Jenifer Fernández Martínez, Lic ${ }^{1(A, B, C, D, E)}$ y Vladimir Martínez Bello, Ph.D.2(A,B,C,D,E) \\ vladimir.martinez@uv.es \\ ${ }^{1}$ Investigadora Independiente, Valencia, España \\ ${ }^{2}$ Facultad de Educación, Universitat de Valencia, España
}

Envío original: 06/08/2016. Reenviado: 28/10/2016, 16/11/2016

Aceptado: 30/11/2016. Publicado: 24/12/2016

Doi: http://dx.doi.org/10.15517/pensarmov.v14i2.25863

\section{RESUMEN}

Fernández-Martínez, J. \& Martínez-Bello, V. (2017). Análisis exploratorio y comparativo de los niveles de actividad física en sesiones de motricidad en educación infantil. PENSAR EN MOVIMIENTO: Revista de Ciencias del Ejercicio y la Salud, 14(2), 1-16. El objetivo del presente estudio consistió en analizar los niveles de actividad física, la composición del grupo y el papel de la maestra durante una sesión tradicional y una sesión alternativa de motricidad infantil en niñas y niños de cinco años de un colegio de la provincia de Valencia, España. Se trató de un estudio descriptivo-exploratorio que utilizó dos instrumentos de recogida de información: un sistema observacional y un sistema de podometría. Participaron ocho sujetos que fueron monitorizados durante las dos sesiones. Los resultados indican que, en la sesión tradicional, la cual estuvo centrada en el juego libre, se observó una mayor presencia de comportamientos sedentarios realizados principalmente en grupo con adulto, grupo 1-1 y solitario, mientras que la conducta del profesorado fue de observadora. Por el contrario, en la sesión alternativa vinculada a un cuento motor, las niñas y niños se mostraron mucho más activos participando en grupos mixtos, y la conducta del profesorado fue la promoción y la demostración de la actividad física. Además, se observó una diferencia en el recuento de pasos entre las dos sesiones. En conclusión, incluso una sesión de menor

-1 -

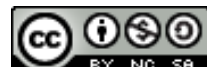


tiempo, pero estructurada a través de un cuento motor, es capaz de promover niveles más altos de actividad física. Dado su carácter exploratorio, este trabajo espera contribuir a la discusión en el ámbito iberoamericano de cómo se desarrollan las clases de motricidad y cuáles son los determinantes de la actividad física en la primera infancia. Son necesarios futuros estudios que profundicen sobre estas temáticas.

Palabras claves: Actividad física; motricidad infantil; juego libre; sistema observacional.

\begin{abstract}
Fernández-Martínez, J. \& Martínez-Bello, V. (2017). Exploratory and Comparative Analysis of the Physical Activity Levels on Motor Performance in Children. PenSAR EN MOVIMIENTo: Revista de Ciencias del Ejercicio y la Salud, 14(2), 1-16. The aim of this study was to analyze the physical activity levels, the group conformation and the teacher's role comparing a traditional session to an alternative motor skill session in five- year old children in Valencia (province in Spain.) This exploratory-descriptive study assessed two different instruments to gather information: observation system and pedometer method. During two sessions, eight subjects were monitored. The results of the study indicated that, during the traditional session focused on free activities, more sedentary behaviors were observed especially when an adult was present, group 1-1 and solitaire, while the teacher role was of observer. On the contrary, in the alternative session based on a physical story, both girls and boys were more active and participated in mixed groups while teacher's behavior was more active promoting and showing how to achieve the physical activity. Furthermore, between the two sessions a difference in the step recounting was observed. Since this is an exploratory study, what we pretend is to contribute to the discussion in the Ibero- American states to evaluate how motor skills can be applied and discriminate the determiners of physical activity in childhoods. Future studies will be necessary to gain in depth knowledge about this topic.
\end{abstract}

Key Words: physical activity, childhood motor skills, free activity, observation system.

\title{
RESUMO
}

Fernández-Martínez, J. \& Martínez-Bello, V. (2017). Análise exploratória e comparativa dos níveis de atividade física em sessões de motricidade em educação infantil. PENSAR EN movimiento: Revista de Ciencias del Ejercicio y la Salud, 14(2), 1-16. O objetivo do presente estudo consistiu em analisar os níveis de atividade física, a composição do grupo e o papel da professora durante uma sessão tradicional e uma sessão alternativa de motricidade infantil nas meninas e meninos de cinco anos de um colégio da província de Valência, Espanha. Tratou-se de um estudo descritivo-exploratório que utilizou dois instrumentos de coleta de informação: um sistema observacional e um sistema de podometria. Participaram oito indivíduos que foram monitorados durante as duas sessões. Os resultados indicam que, na sessão tradicional, a qual esteve centralizada no jogo livre, observou-se uma maior presença de comportamentos sedentários realizados principalmente no grupo com adulto, grupo 1-1 e solitário; por outro lado, a conduta do professorado foi de observadora. Pelo contrário, na sessão alternativa vinculada a um conto motor, as meninas e meninos se mostraram muito mais ativos participando em grupos mistos, e a conduta do professorado foi a promoção e a demonstração da atividade física. Além disso, observou-se uma diferença na recontagem de passos entre as duas sessões. Em conclusão, inclusive uma sessão de menor tempo, mas estruturada através de um conto motor, é capaz de promover níveis mais altos de atividade física. Dado seu caráter exploratório, este trabalho 
espera contribuir com a discussão no âmbito ibero-americano sobre como se desenvolvem as aulas de motricidade e quais são as determinantes da atividade física na primeira infância. São necessários futuros estudos que perquiram sobre estas temáticas.

Palavras-chaves: Atividade física; motricidade infantil; jogo livre; sistema observacional.

La escuela infantil (3-6 años) se encuentra en una posición privilegiada para promover la actividad física (AF) con el objetivo de disminuir los niveles de obesidad infantil y combatir la tendencia de bajos niveles de AF presentes en esta etapa educativa (Lu y Montague, 2015). En este sentido, se ha encontrado que sesiones regulares de AF por semana pueden promover importantes adaptaciones fisiológicas relacionadas con el bienestar integral de niñas y niños de edad preescolar (Timmons, Naylor y Pfeiffer 2007).

En este marco, la Organización Mundial de la Salud (2010) establece que una AF moderada-vigorosa (AFMV) superior a 60 minutos al día genera beneficios adicionales sobre la salud. A su vez, el Instituto Nacional del Deporte y la Educación Física de Estados Unidos recomienda que los menores de seis años inviertan como mínimo 60 minutos de AFMV realizada a través de actividades estructuradas y 60 minutos de AF no estructurada al día (Clark et al., 2002). No obstante, Tucker (2008) en una revisión de literatura, encontró que solo el $54 \%$ de los preescolares cumplieron con las recomendaciones de AF. Recientemente, en el ámbito español, Torres-Luque, Beltrán, Calahorro, López-Fernández y Nikolaidis (2016) encontraron que el $88,8 \%$ de niñas y niños de 4 años no cumplieron con las recomendaciones de $120 \mathrm{~min} /$ día. Por esta razón, como señalan distintos autores, el profesorado en educación infantil (EI) se encuentra en una posición única para apoyar y motivar estilos de vida activos en la primera infancia (Eastman, 1997; Froehlich y Humbert, 2011), que se pueden manifestar como hábitos adquiridos en años posteriores (Biddle, Pearson, Ross y Braithwaite, 2010).

Estudios a nivel internacional y en el ámbito regional europeo señalan bajos niveles de AFMV y altos niveles de comportamientos sedentarios en niñas y niños de El. A nivel internacional, Chow, McKenzie y Louie (2015), en un estudio con niñas y niños de educación preescolar en Hong Kong, encontraron que los niveles de AFMV se modificaron según el contexto de la sesión, en donde se observó que las niñas y los niños estuvieron más tiempo en AFMV cuando la actividad estuvo centrada en el desarrollo de las habilidades motrices. En los Estados Unidos, Wadsworth, Rudisill, Hastie, Boyd y Rodríguez-Hernández (2014) compararon los niveles de AF de niñas y niños de 4 años entre una sesión estructurada de educación física (EF) y una sesión no estructurada centrada en el juego libre. Encontraron que los preescolares permanecieron un $86 \%$ del tiempo en actividades sedentarias en la sesión no estructurada comparada con la sesión estructurada que correspondió a un 31\%. Recientemente, Palmer, Matsuyama y Robinson (2016), al comparar los niveles de AF en niñas y niños de El durante dos sesiones, encontraron menos comportamientos sedentarios en la sesión de movimiento estructurado comparado con una sesión de juego libre al aire libre. Observaron que en la sesión no estructurada el porcentaje del tiempo en actividades sedentarias fue del $80 \%$ mientras que en la sesión estructurada alcanzó el 50\%. A nivel regional europeo, Van Cauwenberghe, Labarque, Gubbels, De Bourdeaudhuij y Cardon (2012) encontraron que el 54\% del tiempo 
de duración de las sesiones de EF se presentaron comportamientos sedentarios, mientras que solo la tercera parte del tiempo los participantes realizaron AFMV.

En el ámbito iberoamericano se han realizado estudios con el objetivo de conocer los siguientes aspectos: la presencia de la motricidad infantil, según la frecuencia y la duración en el ámbito de la El desde el reporte de profesionales en formación (Pons-Rodríguez y Arufe-Giráldez, 2016); determinar las opiniones del profesorado en El sobre el papel de la psicomotricidad (Martín-Domínguez y Soto-Rosales, 2009); analizar la intervención docente en el ámbito de la psicomotricidad en escuelas infantiles a través de encuestas (Moreno, López, Gutiérrez, Cascada y Rosario-Fernández, 2004; Viscarro, Gimeno y Antón, 2012; Simó-Llobet y Espada-Mateos, 2013). También se han propuesto sistemas de observación psicomotriz para evaluar los progresos del estudiantado (De Andrés-Rubio y GarcíaArroyo, 1993; Herrán-Izaguirre, 2006) y se han determinado las diferencias entre distintos métodos de intervención en psicomotricidad, en relación con el desarrollo psicomotor (Jiménez-Díaz y Araya-Vargas, 2010; Terry-Andrés, 2014).

No obstante lo anterior, para quienes escribimos este artículo no existe evidencia en el ámbito iberoamericano sobre la evaluación de los niveles y patrones de AF durante sesiones de motricidad infantil a través de sistemas objetivos y observacionales en niñas y niños del segundo ciclo de El (3-6 años). En este sentido, se considera que la apertura de canales de comunicación entre países que comparten la lengua castellana para el intercambio de programas y aplicaciones didácticas vinculadas con la AF puede ser una oportunidad para debatir y construir alternativas que mejoren la calidad de vida de las niñas y niños menores de 6 años. Por tanto, el objetivo del presente estudio consistió en analizar los niveles de AF, la composición del grupo y el papel de la maestra durante una sesión tradicional y una sesión alternativa de motricidad infantil en niñas y niños de cinco años de un colegio de la provincia de Valencia, España.

\section{METODOLOGÍA}

Diseño. Se trató de un estudio descriptivo-exploratorio de carácter comparativo. El estudio fue realizado en un aula de El de 5 años de un colegio público de la provincia de Valencia, España. Tuvo una duración de cuatro meses. Se realizaron las gestiones oportunas para solicitar permiso a las autoridades competentes, a las niñas y niños, y finalmente a las madres y padres del estudiantado. Todas las familias dieron su consentimiento.

Grupo clase. El grupo de clase estuvo formado por 25 estudiantes: 13 niños y 12 niñas de tercer curso del segundo ciclo de educación de El, con una edad promedio de $5.4 \pm$ 0.6. En el centro escolar la sesión de motricidad se realiza una vez por semana. La duración de la misma es de 90 minutos y es realizada por la maestra titular del grupo.

Participantes. Del grupo de clase, para el análisis del sistema observacional y de la podometría se seleccionaron ocho sujetos (Tabla 1). Se procedió a realizar una selección por conveniencia. En las semanas previas al inicio del estudio, después de observar el comportamiento cotidiano en las sesiones de motricidad y en el horario tradicional, se seleccionaron dos niñas y dos niños con una actitud más proclive al movimiento, y dos niñas y dos niños con una actitud más pasiva. Fueron cuatro niñas (P1, P2, P3 y P4) y cuatro niños (P5, P6, P7 y P8). Se tomaron los datos de talla y peso y se calculó el índice de 
masa corporal (IMC) siguiendo el documento Patrones de Crecimiento de la Organización Mundial de la Salud (2006). Cinco participantes fueron identificados con peso normal, dos con bajo peso y uno con sobrepeso.

Tabla 1

Datos de talla, peso e IMC de las niñas y niños participantes

\begin{tabular}{ccccc}
\hline & \multicolumn{2}{c}{ Niñas } & \multicolumn{2}{c}{ Niños } \\
\cline { 2 - 5 } & Media & DT & Media & DT \\
\hline Talla & 113 & 4.2 & 116 & 3.4 \\
Peso & 18.6 & 2.2 & 21.7 & 4.3 \\
IMC & 14.4 & 1.0 & 15.8 & 2.2 \\
\hline
\end{tabular}

Nota: IMC, índice de masa corporal; DT, desviación típica. Fuente: elaboración propia.

Instrumentos de medición de la AF. El primer instrumento, de carácter cuantitativo, estuvo destinado a determinar el número de pasos a través de un sistema de podometría. El segundo, de carácter cualitativo, estuvo destinado a determinar la presencia de comportamientos sedentarios, de AF moderadas y AFMV, así como el papel del profesorado (Instrumento SOFIT-P). La categoría composición del grupo fue tomada del sistema observacional OSRAC-P (Sistema observacional de registro de la AF en preescolar) (Brown et al., 2006).

Podometría. Para contar el número de pasos, las niñas y niños portaron un podómetro durante las dos sesiones de motricidad infantil. Se utilizaron ocho podómetros Yamax Digiwalker SW-200 (Yamax, 2016). El instrumento utiliza un brazo horizontal tipo péndulo que se mueve hacia arriba y abajo, y cuando se mueve la cadera, se abre y cierra un circuito eléctrico. La podometría ha sido empleada en el ámbito de la El y su relación con la AF. McKee, Boreham, Murphy y Nevill (2005) validaron el recuento de pasos a través de un sistema de podometría frente a los valores reportados por un sistema observacional.

Sistema de observación. Para analizar los niveles de AF y el papel de la maestra durante las sesiones se procedió a utilizar el sistema de observación SOFIT-P (Sistema de observación del tiempo de instrucción física en preescolares), el cual es una versión ajustada del SOFIT. EI SOFIT-P es un instrumento construido originalmente por McKenzie, Sallis y Nader (1991) y utilizado en el ámbito de la El para evaluar los determinantes de la AF (Sharma, Chuang, Skala y Atteberry, 2011). Respecto a la categoría Nivel de AF los indicadores fueron: Comportamientos sedentarios (Sentado, acostado, en pie, etc.), Caminando y Muy activo (Galopar, saltar, correr, etc.). En relación a la categoría Papel de la maestra, los indicadores fueron: Promueve, Demuestra, Orienta, Administra, Observa y Otros. Para analizar la composición el grupo, esta categoría fue tomada del instrumento OSRAC-P (Brown et al., 2006). Los indicadores fueron: Solitario, 1-1, Grupo adulto, Grupo niñas, Grupos niños y Grupo mixto. Una observadora ajena al estudio y la investigadora del estudio recibieron entrenamiento en la lectura y captación de datos siguiendo el sistema SOFIT-P durante tres semanas (McKenzie, 2002; Sharma et al., 2011) con discusión posterior para el esclarecimiento de las dudas. El entrenamiento se realizó en tres momentos. 
En un primer momento, el grupo observacional fue reunido para explicar los objetivos y la metodología. En un segundo momento, el grupo realizó una prueba en el aula de motricidad con una plantilla de registro. El aula de motricidad tuvo suelo de goma y estuvo provista de material didáctico fijo y no fijo. Destaca como material fijo las espalderas, pizarra, etc., y como material no fijo elementos tales como cuerdas, bancos, conos, pelotas, etc. Finalmente, el registro de los datos fue verbalizado entre los miembros del grupo para describir aquellas conductas que necesitaban ser clarificadas.

Procedimientos. Después de recibir entrenamiento en los dos sistemas se llevó a cabo la sesión tradicional. Dos semanas después se procedió a realizar la sesión alternativa. La forma de recolectar la información en las dos sesiones de motricidad fue la siguiente: Dos minutos antes de inicio de la sesión se colocó en la cintura (parte lateral derecha) el podómetro, que fue retirado inmediatamente terminada la sesión. Durante el desarrollo de la sesión, cada participante fue identificado y se procedió a aplicar el sistema de observación por el equipo observador entrenado para tal efecto. Una vez recogidos los datos fueron incorporados a una hoja de cálculo para su posterior análisis.

Sesiones de motricidad para valorar la AF. Se utilizaron dos sesiones de motricidad infantil. Una sesión tradicional y una sesión alternativa. En las dos sesiones participó todo el grupo. La determinación de los niveles de AF a través de podometría y el sistema observacional en las dos sesiones se realizó con los mismos ocho sujetos.

Sesión tradicional. Las clases de motricidad se desarrollan una vez por semana, tienen una duración de 90 minutos y se basan principalmente en el juego libre. Antes de iniciar la sesión se reparten los materiales en el espacio del salón. La sesión se estructura normalmente en tres momentos y en el momento de despedida se procede a realizar un dibujo sobre la sesión. Esta misma estructura se siguió en la evaluación de la sesión.

Sesión alternativa. Una vez recogida la información de la sesión tradicional se decidió construir una sesión alternativa. Para ello, se consensuó con la maestra una unidad didáctica de seis sesiones de 45 minutos alrededor del tema "Perdidos en la Selva". Para la observación, se construyó particularmente una sesión de motricidad siguiendo la propuesta de Vaca-Escribano (2000) de tres momentos. La parte inicial contó con tres actividades, donde se potencia el juego cooperativo y la coeducación, a la vez que se realiza una puesta a punto para la AF. La segunda parte de la sesión consistió en un cuento motor donde la docente participa con el alumnado. Durante la última parte de la sesión se desarrolló un cuento con pictogramas. Tras finalizar la sesión, realizaron un dibujo de lo que más les había gustado de la sesión. Se consideró que relacionar un tema como la selva es una forma de trabajar y potenciar los niveles de AF desde una perspectiva más atractiva tanto para las niñas como los niños. En la sesión alternativa participó la totalidad del grupo (25 niños y niñas), de los cuales 8 participaron en la observación y portaron el podómetro como fue explicado previamente.

Análisis estadísticos. Se realizó un análisis descriptivo teniendo en cuenta los porcentajes de las observaciones recogidas a través del SOFIT-P. Se utilizó la correlación de Pearson con el objetivo de evaluar la relación entre el número de pasos y los niveles de 
AF observados con el sistema SOFIT-P y el IMC. Respecto a los datos de podometría se utilizó la media y la desviación típica como medidas de tendencia central. Para establecer una diferencia entre el recuento de pasos en la sesión tradicional y la sesión alternativa se utilizó la prueba $t$-Student para muestras dependientes. Se asumió una diferencia estadísticamente significativa con un valor de probabilidad menor de 0.05 . Los datos fueron analizados con el programa estadístico SPSS (Versión 21).

\section{RESULTADOS}

Para facilitar la lectura de los resultados y su posterior discusión, se presenta la comparación de las sesiones (tradicional - alternativa) en cada medición realizada por el recuento de pasos, el SOFIT-P y el OSRAC-P.

Recuento de pasos. En la Tabla 2, se observa que el recuento de pasos en la sesión alternativa fue superior a la alcanzada en la sesión tradicional. Al aplicar el estadístico $t$ Student se observó una diferencia significativa $(t=-3.497, g l=7 ; p=0.01)$.

Relación entre el número de pasos y los niveles de AF en SOFIT-P. Se observó una correlación negativa entre el número de pasos y las observaciones de cada sujeto de acuerdo al indicador comportamientos sedentarios y una correlación positiva entre el número de pasos y las observaciones de cada sujeto de acuerdo a los indicadores caminando $(r=-7.82 ; p=0.022)$ y muy activo $(r=0.775 ; p=0.024)$. No se observó ninguna correlación entre el número de pasos y el IMC $(r=0.132 ; p=0.756)$.

Tabla 2

Recuento de pasos

\begin{tabular}{ccc}
\hline & $\begin{array}{c}\text { Sesión } \\
\text { tradicional }\end{array}$ & $\begin{array}{c}\text { Sesión } \\
\text { alternativa }\end{array}$ \\
\hline P 1 & 1.046 & 1.838 \\
P 2 & 563 & 1.952 \\
P 3 & 1.359 & 2.339 \\
P 4 & 2.039 & 2.432 \\
P 5 & 1.123 & 1.925 \\
P 6 & 1.929 & 2.147 \\
P 7 & 1.321 & 1.429 \\
P 8 & 1.809 & 1.845 \\
\hline Media & $1.399\left(^{*}\right)$ & 1.988 \\
D.T. & 502 & 318
\end{tabular}

Nota: P, Participante. D.T., Desviación típica. $\left({ }^{*}\right) p<0.05$ entre Sesión tradicional vs. Sesión alternativa. Fuente: elaboración propia.

Niveles de AF (SOFIT-P) individuales. En la Figura 1 se observan los porcentajes de presencia de la distribución de los tres niveles de AF durante la sesión tradicional y alternativa. De manera descriptiva, todos los participantes en la sesión tradicional presentan 
porcentajes entre el $50 \%$ y $90 \%$ del indicador comportamientos sedentarios, mientras que los niveles de caminando y muy activo se encuentran entre el 5 - $40 \%$ y $0-40 \%$ respectivamente. Por el contrario, en la sesión alternativa, los comportamientos sedentarios se distribuyen entre el $0 \%$ y el $50 \%$. Es de notar que dos sujetos (número 4 y 8 ) presentaron un $75 \%$ y un $65 \%$ de este indicador, respectivamente. Los porcentajes de los indicadores caminando y muy activo se distribuyeron entre el $15-75 \%$ y $0-60 \%$, respectivamente.

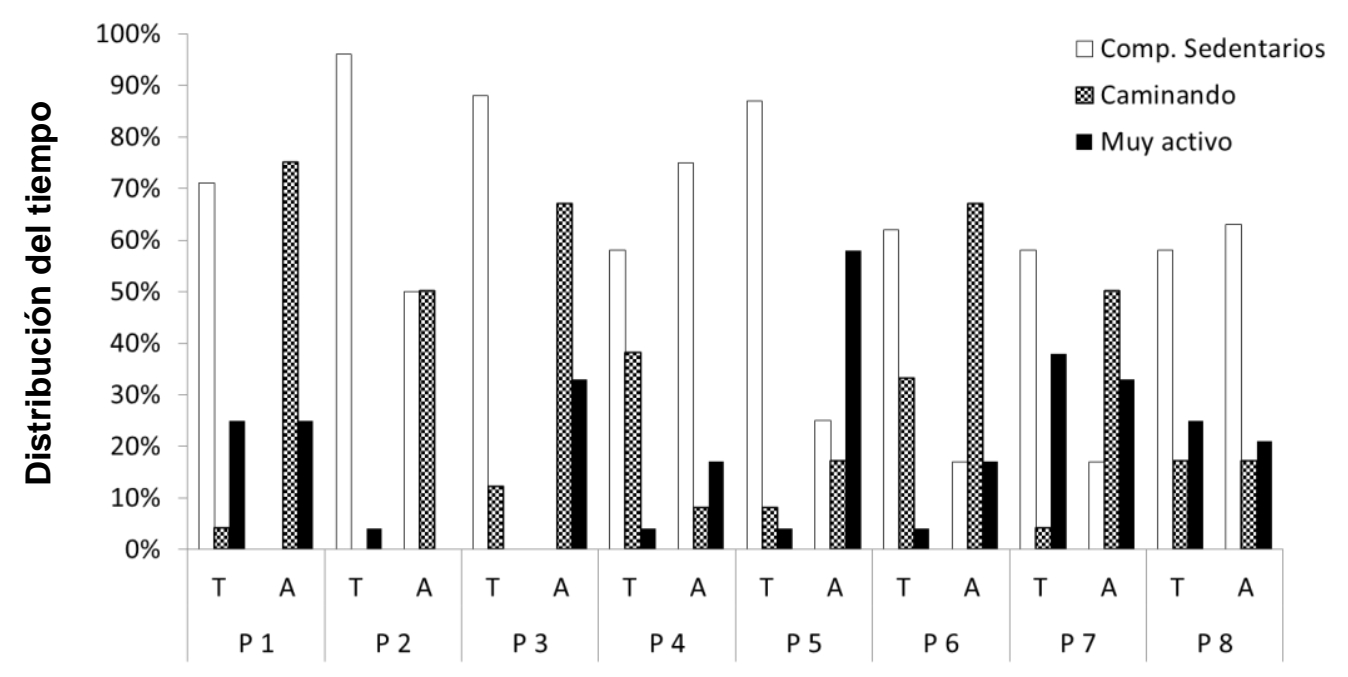

Participantes en las dos sesiones

Figura 1. Distribución del tiempo de AF de niñas y niños durante la sesión tradicional (T) y la sesión alternativa (A). Nota: T, sesión tradicional; A, sesión alternativa; Comp., Comportamientos; $P$, Participantes. Fuente: elaboración propia.

Niveles de AF (SOFIT-P) grupales. En la Tabla 3 se observa que en la sesión tradicional en el $72 \%$ de las observaciones se presentaron comportamientos sedentarios. Por el contrario, durante la sesión alternativa, los comportamientos sedentarios representaron el $30 \%$ de las observaciones mientras que los indicadores caminando y muy activo correspondieron al $44 \%$ y $26 \%$, respectivamente.

Papel de la maestra (SOFIT-P). En la Tabla 4, respecto al papel de la maestra durante las sesiones, se observa que mientras que en la sesión tradicional la conducta con mayor porcentaje fue observar $(60 \%)$, esta conducta en la sesión alternativa solo tuvo una presencia de $8 \%$. A su vez, la demostración y la promoción de la AF tuvieron una presencia importante en el total de observaciones de la sesión alternativa. En la sesión tradicional, las conductas de demostración y promoción fueron nada y poco presentes, respectivamente. 
Tabla 3

Promedio de los porcentajes de observaciones de los niveles de AF durante las sesiones

\begin{tabular}{cccc}
\hline & \multicolumn{3}{c}{ Nivel de $A F$} \\
\cline { 2 - 4 } & $\begin{array}{c}\text { Comportamientos } \\
\text { sedentarios }\end{array}$ & Caminando & Muy activo \\
\hline Sesión tradicional & 72 & 15 & 13 \\
Sesión alternativa & 30 & 44 & 26 \\
\hline
\end{tabular}

Fuente: elaboración propia.

Tabla 4

Porcentaje de observaciones de acuerdo a las categorías papel de la maestra durante las sesiones

\begin{tabular}{ccccccc}
\hline & \multicolumn{5}{c}{ Papel de la maestra } \\
\cline { 2 - 7 } & Promueve & Demuestra & Orienta & Administra & Observa & Otros \\
\hline $\begin{array}{c}\text { Sesión } \\
\text { tradicional } \\
\begin{array}{c}\text { Sesión } \\
\text { alternativa }\end{array}\end{array}$ & 29 & 1 & 3 & 16 & 60 & 20 \\
\hline
\end{tabular}

Fuente: elaboración propia.

Composición del grupo (OSRAC-P). Respecto a la categoría composición del grupo, en las dos sesiones se observa que el grupo adulto es el indicador con mayor presencia (Tabla 5). A su vez, en la sesión tradicional se observaron interacciones de grupo de niñas, relaciones 1-1 y realización de actividades individuales, composiciones que no fueron observadas en la sesión alternativa. En esta última, alrededor de la mitad de las observaciones se observó la composición de grupo mixto, mientras que en la sesión tradicional esta no superó el $6 \%$.

Tabla 5

Porcentaje de observaciones de acuerdo a la categoría composición del grupo durante las sesiones

\begin{tabular}{ccccccc}
\hline & \multicolumn{6}{c}{ Composición del grupo } \\
\cline { 2 - 7 } & Solitario & $1-1$ & $\begin{array}{c}\text { Grupo } \\
\text { adulto }\end{array}$ & $\begin{array}{c}\text { Grupo } \\
\text { niñas }\end{array}$ & $\begin{array}{c}\text { Grupo } \\
\text { niños }\end{array}$ & $\begin{array}{c}\text { Grupo } \\
\text { mixto }\end{array}$ \\
\hline $\begin{array}{c}\text { Sesión } \\
\text { tradicional } \\
\begin{array}{c}\text { Sesión } \\
\text { alternativa }\end{array}\end{array}$ & 14 & 16 & 40 & 18 & 6 & 6 \\
\hline
\end{tabular}

Fuente: elaboración propia. 


\section{DISCUSIÓN}

Una desventaja de los sistemas cuantitativos de medición de la AF es que no ofrecen información sobre el tipo y el contexto en el que esta se desarrolla (Soini et al., 2014; Pate et al., 2014). Sin embargo, como señalan McKenzie y Van der Mars (ㅁ15) la observación sistemática (OS) supera con creces otras medidas de la AF al identificar los contextos sociales en los cuáles esta ocurre. Por tanto, en el presente estudio y con el objetivo de conocer los niveles de AF en sesiones de motricidad infantil, se utiliza por primera vez en el ámbito iberoamericano la información aportada por un sistema cualitativo (SOFIT-P y OSRAC-P) y un sistema cuantitativo de medición de la AF (Podometría).

Los niveles de AF durante las sesiones. Respecto a la sesión tradicional, los resultados señalan que las niñas y niños realizaron durante gran parte del tiempo comportamientos sedentarios (Tabla 3 ). Similar resultado ha sido observado en niñas y niños belgas. Van Cauwenberghe et al. (2012) encontraron que el 54\% del tiempo de duración de las sesiones de EF se presentaron comportamientos sedentarios, mientras que solo la tercera parte del tiempo las niñas y niños participaron en actividades de intensidad moderada-vigorosa. En los Estados Unidos, Wadsworth et al. (2014) encontraron que los preescolares permanecieron un mayor porcentaje del tiempo en actividades sedentarias en la sesión no estructurada comparada con la sesión estructurada (36\% en AFMV en la sesión estructurada mientras que en la sesión no estructurada no se observó ninguna AFMV).

Por el contrario, durante la sesión estructurada se presentaron más observaciones de los indicadores (caminando y muy activo), tanto en niñas como en los niños (Figura 1). A su vez, el recuento de pasos se modificó de manera estadísticamente significativa en la sesión alternativa comparada con la sesión tradicional (Tabla 2). Al no contar con estudios en el ámbito iberoamericano, esta parte de la discusión se centrará en estudios en el ámbito anglosajón. Recientemente, Palmer et al. (2016) compararon los niveles de AF en niñas y niños de El durante dos oportunidades diferentes de AF: juego libre el aire libre y una sesión de movimiento estructurada. Las autoras encontraron menos comportamientos sedentarios en la sesión de movimiento estructurado comparada con la del juego libre con la del aire libre. Al mismo tiempo, se observó que el 50\% del tiempo, a diferencia de la sesión de juego libre, las niñas y los niños estuvieron participando en AF saludables. Por tanto, se comparte la apreciación de Wadsworth et al. (2014) en el sentido de que las sesiones de EF estructuradas basadas en un clima motivacional de calidad están en capacidad de aumentar los niveles de AFMV en el ámbito de la El.

Recientemente, De Craemer et al. (2015) trasladaron las recomendaciones de AF ligera y AFMV medidas por acelerometría al recuento de pasos diarios en niñas y niños de educación infantil. Encontraron que para cumplir con los 120 minutos de AF ligera y AFMV mínima al día se deben alcanzar aproximadamente 11.500 pasos. Por tanto, sin intención de comparar, pero con el objetivo de aportar más información, de los resultados de nuestro estudio se encontró que en la sesión alternativa se reportaron en promedio 1988 pasos. De esta manera, la clase de motricidad aportaría alrededor del $18 \%$ de la AFMV diaria. Desconocemos la cantidad de AFMV que aportan otros espacios (como por ejemplo el periodo de descanso en el patio de recreo), pero teniendo en cuenta que esta sesión solo se realiza una vez por semana, se necesitarían muchas más opciones de AF, estructurada 
o no, para aumentar estos niveles, al menos en este centro y en esta aula. Desde una perspectiva complementaria, Jiménez-Díaz y Araya-Vargas (2010), en un estudio que estuvo dirigido a evaluar el efecto de distintas sesiones estructuradas sobre las habilidades motrices, encontraron una mejora significativa en la habilidad locomotora de galopar solamente en el grupo de 90 minutos, comparada con una de 60 y 30 minutos, respectivamente. Sin embargo, la sesión de este último estudio de 90 minutos utilizó una metodología de enseñanza de resolución de problemas y descubrimiento dirigido, basadas en juegos. Con base en los resultados del presente estudio, se concluye que las sesiones estructuradas son importantes para aumentar los niveles de AF y, como apuntan JiménezDíaz y Araya-Vargas (2010), para mejorar las habilidades motrices locomotoras. En definitiva, de los resultados descriptivos del sistema observacional, así como del aumento significativo en el número de pasos de la sesión alternativa respecto a la sesión tradicional, señalan que incluso una sesión de menor tiempo, pero estructurada a través de un cuento motor, es capaz de promover más altos niveles de AF.

El papel de la maestra y la composición del grupo durante las sesiones. De acuerdo con la categoría participación de la maestra se observa que una participación más activa del personal docente con dos conductas positivas (promover y demostrar) tiene una mayor presencia en la sesión alternativa (Tabla 4). Al inicio de la sesión de juego libre la maestra desplegó los materiales alrededor de la sala y, durante la misma, la conducta con una mayor representación fue de observar. Por el contrario, en la sesión alternativa esta conducta quedó relegada a solo un $8 \%$ de presencia, mientras que otras conductas como demostrar y promover se manifestaron durante la sesión. En particular, de manera descriptiva se puede intuir que la participación de la maestra en la motivación hacia el estudiantado en la sesión alternativa, la demostración de las conductas, esto es, el involucrarse en ellas y la disminución del papel pasivo en la sesión puede ser responsable del aumento en los niveles de AF de las niñas y de los niños. Distintas aproximaciones han sido encontradas en otros estudios. A nivel regional europeo, Van Cauwenberghe et al. (2012) encontraron que la promoción de la AF y la demostración tuvieron una presencia menor del $12 \%$, respectivamente, mientras que la instrucción y la administración de la clase ocuparon el $49 \%$ y el $23 \%$, respectivamente. Las autoras encontraron también una asociación negativa entre el tiempo en AFMV y la implicación del profesorado en la promoción de la AF durante la sesión. Sin embargo, como ellas mismas afirman en su escrito, al ser las sesiones de AF dirigidas y centradas principalmente en el desarrollo de las habilidades motrices, concluyen que existe una relación entre el tiempo en el que profesorado invierte en dar instrucciones y los niveles de AF. Por el contrario, en el presente estudio la sesión alternativa estuvo centrada en un cuento motor con juego de roles y representación. A su vez, el estudio de Van Cauwenberghe et al. (2012) señala que solo el $11 \%$ del tiempo de la sesión estuvo destinada al juego estructurado y un $41.7 \%$ al desarrollo de habilidades motrices. También, Chow et al. (2015) observaron que el tiempo en AFMV estuvo negativamente asociado con el porcentaje del tiempo en que el profesorado invierte en dar instrucciones y gestionar la clase. Por el contrario, encontraron que los niveles de AFMV estuvieron positivamente asociados con el porcentaje del tiempo que el profesorado invierte observando al estudiantado durante la sesión. Con base en los resultados del presente estudio, por el contrario, en la sesión alternativa las conductas de dar instrucciones y observar fueron mínimas, y esto se considera fundamental en que los 
niveles de AF hubiesen aumentado. En definitiva, se considera que en la medida en que se modifica el contexto de la sesión, los intereses, objetivos y respuestas también se ven modificados. En definitiva, el papel de la maestra afecta directamente el nivel de AF en la clase.

Por tanto, puede sugerirse que la incorporación en el currículo de El de sesiones estructuradas de AF dentro la programación diaria del centro puede aumentar los niveles de AF y reducir los comportamientos sedentarios durante la jornada escolar (Van Cauwerberghe et al., 2012). Por otra parte, Terry-Andrés (2014) encontró que las niñas y niños en primer ciclo de El que participaron en sesiones estructuradas bajo una metodología vivencial dirigida obtuvieron una mejora en el desarrollo de las habilidades motrices comparados con aquellos que participaron en sesiones exclusivamente vivenciadas. Sin embargo, como sostienen otros autores (Burdette y Whitaker, 2005), esta incorporación de actividades programadas no puede ser utilizada a expensas de las oportunidades de tiempo de juego libre tan importantes en esta etapa. Bajo esta línea, la metodología alternativa utilizada en el presente estudio podría ser una opción para favorecer la práctica de AF y utilizar el tiempo restante en generar oportunidades de juego libre. Por ejemplo, al reducir de 90 a 45 minutos la duración (como se hizo en la sesión estructurada) se podrían fomentar, de acuerdo con una modificación del currículo del profesorado y del centro, otras oportunidades de juego libre activo. De igual forma, como señala Burdette y Whitaker (2005) son las oportunidades de juego libre activo aquellas que están en posibilidad de promover la AF y fomentar la formación integral de la niña y del niño. Por tanto, no es el juego libre per se quien promueve altos niveles de sedentarismo, sino la repetida presencia de oportunidades de juego libre no activos vinculados normalmente al uso de materiales didácticos, así como en instalaciones bajo techo, que promueven comportamientos sedentarios. Estos resultados demuestran que las intervenciones de AF en el aula de El pueden ser implementadas con éxito usando enfoques flexibles de acuerdo con las necesidades e intereses de cada aula y proyecto curricular (Howie et al., 2016).

Por otra parte, en la sesión alternativa se observó una frecuencia de composición del grupo repartida entre grupo mixto y grupo con adulto, frecuencias sobre estos indicadores menores en la sesión tradicional. Se considera que el trabajo cooperativo realizado durante la sesión alternativa podría explicar más situaciones de construcción de grupo y menos actividades individuales y de grupo (1-1) en la sesión tradicional que podrían estar vinculados con menores niveles de AF. Sin embargo, es una apreciación que debe ser determinada en estudios posteriores.

Limitaciones y perspectivas de futuro. Una fortaleza de este estudio ha sido que se llevó a cabo un diseño de medidas repetidas a través de dos sistemas de recolección de información.

Al tratarse de un estudio descriptivo-exploratorio se presentan las siguientes limitaciones. Se desconoce la relación entre cada una de las categorías, por ejemplo, entre el tipo de sesión y cómo afectan, por ejemplo, los niveles de AF según el sexo. Debido al reducido número de participantes, los resultados no pueden ser extrapolados a otras aulas de El. Son necesarios futuros estudios con más participantes. Finalmente, al no contar con un análisis estadístico inferencial que nos permita encontrar relaciones de causa-efecto, se 
desconocen en profundidad los determinantes del aumento de los niveles de AF en la sesión alternativa.

Sin embargo, la intención del trabajo es que se convierta en un punto de partida para discutir en el ámbito iberoamericano cómo se desarrollan las clases de motricidad infantil y cuáles son los determinantes de la AF en la primera infancia. Por esta razón, se considera que los resultados del presente trabajo de investigación son robustos porque describen los niveles y patrones de la AF a través de la combinación de dos sistemas de recogida de información para esclarecer con mayor precisión cómo se desarrolla la conducta de la AF en el ámbito de la primera infancia.

Finalmente, y en aras de garantizar una perspectiva integral en el análisis de la formación integral de las niñas y los niños, futuros estudios podrían centrarse en indagar sobre variables de bienestar físico y mental, así como en evaluar como en cada sesión interactúan la AF y el desempeño motor, a la par que se conocen los determinantes didácticos y sociales que regulan la promoción de la AF en el ámbito de El.

\section{CONCLUSIONES}

Los resultados obtenidos en el nivel de AF indican que por lo general en la sesión alternativa las niñas y los niños se mostraron mucho más activos participando en grupos mixtos y que la conducta del profesorado fue la promoción de la AF y la demostración, a diferencia de la sesión tradicional. Por tanto, como sostienen Gil-Madrona, Gómez-Víllora, Contreras-Jordán y Gómez-Barreto (2008), unas clases y una cantidad de AF apropiadas en la El desde una perspectiva interdisciplinaria y globalizadora, basada en la acción y en la experimentación, contribuirán al desarrollo cognitivo, físico y social de las niñas y de los niños. Por esta razón, desde la primera infancia se debe promover la AF con el objetivo de concienciar a los más pequeños y hacer que adquieran hábitos saludables para la vida presente y la vida adulta. Finalmente, todos estos resultados implican una mayor responsabilidad por parte del profesorado de El.

\section{REFERENCIAS}

Biddle, S. J., Pearson, N., Ross, G. M., \& Braithwaite, R. (2010). Tracking of sedentary behaviours of young people: a systematic review. Preventive Medicine, 51(5), 345351. doi: https://doi.org/10.1016/i.ypmed.2010.07.018

Brown, W. H., Pfeiffer, K. A., Mclver, K. L., Dowda, M., Almeida, J. M., \& Pate, R. R. (2006). Assessing preschool children's physical activity: the Observational System for Recording Physical Activity in children-preschool version. Research quarterly for exercise and sport, 77(2), 167-176. Recuperado de http://www.tandfonline.com/ doi/abs/10.1080/02701367.2006.10599351

Burdette, H. L., y Whitaker, R. C. (2005). Resurrecting free play in young children: looking beyond fitness and fatness to attention, affiliation, and affect. Archives of Pediatrics \& Adolescent Medicine, 159(1), 46-50. Recuperado de https://doi.org/10.1001/ archpedi.159.1.46

Chow, B. C., McKenzie, T. L., y Louie, L. (2015). Children's Physical Activity and Associated Variables during Preschool Physical Education. Advances in Physical Education, 5, 39-49. Recuperado de https://doi.org/10.4236/ape.2015.51005 
Clark, J. E., Clements, R. L., Guddemi, M., Morgan, D. W., Pica, R., Pivarnik, J., Rudisill, M., Small, E., y Virgilio, S. (2002). Active start: A statement of physical activity guidelines for children birth to five years. Reston, United States of America: National Association for Sport and Physical Education.

De Andrés Rubio, M. N., y Arroyo, M. J. G. (1993). La observación psicomotriz como técnica de evaluación en la etapa de la Educación Infantil. Aula: Revista de Pedagogía de la Universidad de Salamanca, (5), 209-222. Recuperado en https://dialnet.unirioja.es/servlet/articulo?codigo $=122483$

De Craemer, M., De Decker, E., De Bourdeaudhuij, I., Verloigne, M., Manios, Y., y Cardon, G. (2015). The translation of preschoolers' physical activity guidelines into a daily step count target. Journal of Sports Sciences, 33(10), 1051-1057. doi: https://doi.org/10.1080/02640414.2014.981850

Eastman, W. (1997). Active living: Physical activities for infants, toddlers, and preschoolers. Early Childhood Education Journal, 24(3), 161-164. doi: https://doi.org/10.1007/BF02353273

Froehlich, A., y Humbert, L. (2011). Physical Activity and Nutrition in Early Years Care Centres: Barriers and Facilitators. Canadian Children Journal of the Canadian association for Young children, 36(1), 26-30. Recuperado de http://mmduvic.ca/ index.php/ics/article/viewFile/15149/6031\#page $=26$

Gil-Madrona, P., Gómez-Víllora, S., Contreras-Jordán, O. R., y Gómez-Barreto, I. (2008). Justificación de la educación física en la educación infantil. Educación y educadores, 11(2), 159-177. Recuperado de http://www.scielo.org.co/pdf/eded/ v11n2/v11n2a10.pdf

Herrán-Izagirre, E. (2006). Aprender a saltar. Un estudio de caso. Revista iberoamericana de psicomotricidad y técnicas corporales, (21), 77-90. Recuperado de https://dialnet.unirioja.es/servlet/articulo?codigo $=3735942$

Howie, E. K., Brewer, A. E., Dowda, M., Mclver, K. L., Saunders, R. P., y Pate, R. R. (2016). A Tale of 2 Teachers: A Preschool Physical Activity Intervention Case Study. Journal of School Health, 86(1), 23-30. doi: https://doi.org/10.1111/ josh.12352

Jiménez-Díaz, J., y Araya-Vargas, G. A. (2010). Más minutos de Educación Física en preescolares favorecen el desarrollo motor. Pensar en Movimiento: Revista de Ciencias del Ejercicio y la Salud, 8(1), 1-8. doi: https://doi.org/10.15517/ pensarmov.v8i1.442

Lu, C., y Montague, B. (2015). Move to Learn, Learn to Move: Prioritizing Physical Activity in Early Childhood Education Programming. Early Childhood Education Journal, 44(5). 409-417. Recuperado de http://link.springer.com/article/10.1007/s10643-015$\underline{0730-5}$

Martín-Domínguez, D., y Soto-Rosales, A. (2009). La importancia de los contenidos psicomotores en el contexto educativo: opiniones de los maestros. Investigación en la Escuela, (67), 97-109. Recuperado de http://www.investigacionenlaescuela.es/ articulos/67/R-67 7.pdf

McKee, D. P., Boreham, C. A., Murphy, M. H., y Nevill, A. M. (2005). Validation of the Digiwalker ${ }^{\mathrm{TM}}$ pedometer for measuring physical activity in young children. Pediatric Exercise Science, 17(4), 345-352. doi: https://doi.org/10.1123/pes.17.4.345 
McKenzie, T. L., Sallis, J. F., y Nader, P. R. (1991). System for observing fitness instruction time. J Teach Phys Educ, 11, 195-205. doi: https://doi.org/10.1123/itpe.11.2.195

McKenzie, T. L. (2002). SOFIT (System of Observing Fitness Instruction Time): Overview and Training Manual. San Diego, CA: San Diego State University. Recuperado de http://www.des.ucdavis.edu/faculty/handy/esp178/sofitprotocol.pdf

McKenzie, T. L., y Van Der Mars, H. (2015). Top 10 research questions related to assessing physical activity and its contexts using systematic observation. Research Quarterly for Exercise and Sport, 86(1), 13-29. doi: https://doi.org/10. 1080/02701367.2015.991264

Moreno-Murcia, J. A. M., López, B., Gutiérrez, E. M., Cascada, M., y Rosario-Fernández, M. (2004). Situación actual de la motricidad en la etapa de 0 a 6 años según el profesorado de Educación Infantil. Revista iberoamericana de psicomotricidad y técnicas corporales, (16), 17-34. Recuperado de https://dialnet.unirioja.es/ servlet/articulo?codigo $=3739246$

Organización Mundial de la Salud. (2006). Patrones de crecimiento infantil de la OMS. Métodos y desarrollo. Recuperado de http://www.who.int/childgrowth/standards/es/

Organización Mundial de la Salud. (2010). Recomendaciones mundiales sobre actividad física para la salud. Recuperado de http://apps.who.int/iris/bitstream/10665/ 44441/1/9789243599977 spa.pdf

Palmer, K. K., Matsuyama, A. L., y Robinson, L. E. (2016). Impact of Structured Movement Time on Preschoolers' Physical Activity Engagement. Early Child-hood Education Journal, 1-6. doi: https://doi.org/10.1007/s10643-016-0778-x

Pate, R. R., O'Neill, J. R., Byun, W., Mclver, K. L., Dowda, M., y Brown, W. H. (2014). |Physical activity in preschool children: comparison between Montessori and traditional preschools. Journal of School Health, 84(11), 716-721. doi: https://doi.org/10.1111/josh.12207

Pons-Rodríguez, R., y Arufe-Giráldez, V. (2016). Análisis descriptivo de las sesiones e instalaciones de psicomotricidad en el aula de educación infantil. Sportis. Scientific Journal of School Sport, Physical Education and Psychomotricity, 2(1), 125-146. doi: https://doi.org/10.17979/sportis.2016.2.1.1445

Sharma, S. V., Chuang, R. J., Skala, K., y Atteberry, H. (2011). Measuring physical activity in preschoolers: reliability and validity of the System for Observing Fitness Instruction Time for Preschoolers (SOFIT-P). Measurement in Physical Education and Exercise Science, 15(4), 257-273. doi: https://doi.org/10.1080/1091367X.2011. $\underline{594361}$

Simó-Llobet, A., y Espada-Mateos, M. (2013). La intervención docente en psicomotricidad en los centros de educación infantil de barcelona. Athlos: Revista internacional de ciencias sociales de la actividad física, el juego y el deporte, (5), 55-74. Recuperado de https://dialnet.unirioja.es/servlet/articulo?codigo $=5311417$

Soini, A., Tammelin, T., Sääkslahti, A., Watt, A., Villberg, J., Kettunen, T., Mehtala, A., y Poskiparta., M. (2014). Seasonal and daily variation in physical activity among threeyear-old Finnish preschool children. Early Child Development and Care, 184(4), 589-601. doi: https://doi.org/10.1080/03004430.2013.804070

Tucker, P. (2008). The physical activity levels of preschool-aged children: A systematic review. Early Childhood Research Quarterly, 23(4), 547-558. doi: https://dx.doi.org/ $\underline{10.1016 / \text { j.ecresq.2008.08.005 }}$ 
Terry-Andrés, J. (2014). Análisis de la influencia de la metodología de la intervención psicomotriz sobre el desarrollo de las habilidades motrices en niños de 3 a 4 años (Tesis doctoral). Universidad de Murcia. Recuperado del Repositorio institucional de la universidad de Murcia: https://digitum.um.es/xmlui/handle/10201/40991

Timmons, B. W., Naylor, P. J., y Pfeiffer, K. A. (2007). Physical activity for preschool children-how much and how? Applied Physiology, Nutrition, and Metabolism, 32, 122-134. Recuperado de http://www.nrcresearchpress.com/doi/pdf/10.1139/H07$\underline{112}$

Torres-Luque, G., Beltrán, J., Calahorro, F., López-Fernández, I., y Nikolaidis, P. T. (2016). Análisis de la distribución de la práctica de actividad física en alumnos de educación infantil. Cuadernos de Psicología del Deporte, 16(1), 261-268. Recuperado de http://revistas.um.es/cpd/article/viewFile/254621/192251

Vaca-Escribano, M. (2000). Reflexiones en torno a las posibilidades educativas del tratamiento pedagógico de lo corporal en el segundo ciclo de Educación Infantil. Revista interuniversitaria de formación del profesorado, (37), 103-120. Recuperado de https://dialnet.unirioja.es/servlet/articulo?codigo=118061

Van Cauwenberghe, L., Labarque, V., Gubbels, J., De Bourdeaudhuij, I. y Cardon, G. (2012). Preschooler's physical activity levels and associations with lesson context, teacher's behavior, and environment during preschool physical education. Early Childhood Research Quarterly, 27(2), 221-230. doi: https://doi.org/10.1016/ j.ecresq.2011.09.007

Viscarro, I., Gimeno, S., y Antón, M. (2012). Intervención psicomotriz en el ámbito escolar: Un estudio longitudinal. Revista Española de Educación Física y Deportes, (399), 97-115. Recuperado de http://www.reefd.es/index.php/reefd/article/view/154

Wadsworth, D. D., Rudisill, M. E., Hastie, P. A., Boyd, K. L., y Rodríguez-Hernández, M. (2014). Preschoolers' Physical Activity and Time on Task During a Mastery Motivational Climate and Free Play. MHSalud, 11(1), 26-34. Recuperado de http://www.redalyc.org/articulo .oa?id=237031810004

Yamax (2016). Manual de uso Digi-Walker CW- Series. Serie CW- 200. Japón: Yamax Corporation. Recuperado de http://www.yamaxx.com/digi/cws manual spanish.pdf

Participación: A- Financiamiento, B- Diseño del estudio, C- Recolección de datos, D- Análisis estadístico e interpretación de resultados, E- Preparación del manuscrito. 